\title{
Review \\ Peritoneal Dialysis Related Infection - Peritonitis Management and Prevention
}

\author{
Edwina A Brown DM(Oxon), FRCP \\ Imperial College Renal and Transplant Centre, \\ Hammersmith Hospital, London, UK \\ Email: e.a.brown@imperial.ac.uk
}

PJKD2020;4(supplement 1):29-32

\section{Peritonitis}

Peritonitis is one of the major risks of peritoneal dialysis (PD) causing significant morbidity and in some instances, mortality. It is one of the principal causes of transfer to hemodialysis (HD). Peritonitis rates should be audited by units. The recommended rate by the ISPD is less than 0.5 episodes/year.

\section{Etiology}

There are 2 major routes of infection in the peritoneum:

- skin contaminants;

- from within the peritoneum via the bowel.

Skin contaminants These are introduced at the time of connections or via an infected exit site. S. aureus, MRSA (methicillin resistant $S$. aureus) and $S$. epidermidis (coagulase-negative staphylococcus) are the most common. Staphylococci adhere to the catheter, which can make eradication difficult. This is often the underlying cause of recurrent infections. $S$. aureus usually causes a more severe peritonitis than $S$. epidermidis.

Pseudomonas infections can also occur via skin contamination and are often associated with exit-site infections. They are mostly likely to occur when the exit site has been allowed to get wet.

Gram-negative infections

These are due to bowel organisms introduced into the peritoneum either by poor hygiene or directly from the bowel. Any pathological process increasing bowel permeability can cause peritonitis, most commonly episodes of diarrhoea or diverticulitis. Often mixed organisms are found. Bowel perforation (e.g. appendicitis) should also be considered with mixed Gram-negative peritonitis, especially if bacterial counts are high.

Other types of infection

- Fungal infections can occur following recent prolonged courses of antibiotics.

- TB in immunosuppressed individuals.

- Water-borne atypical mycobacteria very rarely.

\section{Risk Factors and Prevention of Peritonitis}

Risk factors for development of peritonitis

Awareness of and addressing potential risk factors for peritonitis are important to reduce infection rates. It is helpful to consider these as centre-related and patient-related

Patient-related:

- Number of connections and disconnections made each day between catheter or its attached line.

- Poor hand washing.

- Patient ability to carry out connections using sterile non-touch technique.

- Lack of clean area in home or at work to carry out exchanges.

- Poor eyesight unless special connection devices are used or assistance available

- Patients disconnecting themselves from APD machine at night (toilet, care for children, etc.).

- Exit site infections, particularly tunnel infections.

- Diarrhoea, particularly if associated with poor hand washing.

- Diverticular disease, particularly if complicated by diverticulitis.

Centre-related:

- In hospital, exchanges being carried out by poorly trained personnel.

- Frequent use of intraperitoneal antibiotics predisposing to fungal peritonitis.

- Failure to follow protocols for patient training and assessment that they are adequately trained

\section{Prevention of Peritonitis}

Units should consider all the above factors. Prevention is also achieved by:

- patient education and training;

- avoiding constipation - many patients are elderly and will therefore have diverticular disease, and diverticulitis is more common when constipated;

- adequate nurse training with special emphasis on hand washing between patients;

- isolating patients who are carriers of antibiotic-resistant bacteria, such as MRSA or VRE (VRE); 
- ensuring that exchanges are only done by trained personnel when patients are admitted for intercurrent illness or surgery

- reinforcing with patient and family that exchanges at home should only be carried out by family members who have been trained

\section{Clinical Features of Peritonitis}

- Abdominal pain (80\% of patients).

- Cloudy fluid on drainage.

- Fever (50\%).

- Nausea (30\%) and diarrhoea (7 - 10\%).

The severity of an episode of peritonitis depends on the causative organism. The need for hospitalization depends on the severity, and on the ability of the patient to carry out the treatment regimen. No organisms are grown from about 20\% episodes (usually mild). S. epidermidis peritonitis is often clinically mild. Episodes due to $S$. aureus or Gram-negative infections are much more severe and have a worse prognosis.

Abdominal pain is a key symptom but very variable, and in some patients is not a feature until the bags have been cloudy for some days. Patients should be trained to report a cloudy bag as soon as they see one regardless of whether they have any other symptoms. Abdominal pain can be severe and require opioid analgesia. Fever occurs in more severe cases. If there is delay in diagnosis, or failure to respond to treatment, the fluid becomes increasingly turbid and eventually looks like 'pea soup'. Drainage can then become poor as the catheter becomes blocked. Even in milder cases, fibrin can form and block the catheter. Loss of ultrafiltration can be a feature of peritonitis, and may persist even after the episode has cleared.

\section{Complications}

Most cases are mild and respond quickly to antibiotics. Complications occur when the diagnosis is delayed, or there is a poor response to treatment. They include:

- failure to respond, necessitating catheter removal and transfer to HD;

- loss of ultrafiltraton;

- loss of appetite and increased catabolism resulting in malnutrition;

- fungal peritonitis after repeated courses of intraperitoneal antibiotics;

- persistent intra-abdominal sepsis requiring laparotomy and drainage;

- formation of adhesions and later catheter malfunction;

- ileus (in severe infections);

- death (rare).

\section{Diagnosis of Peritonitis}

As shown in Figure 2, the key investigation is microscopy and culture of drained peritoneal dialysate. This should be done as soon as possible after the patient arrives and should only be done by trained personnel. Dialogue with microbiology laboratory is important to maximize culture positivity rates. The fluid sample can be obtained by aspirating with a syringe from a freshly drained bag. The fluid should not be obtained directly from the catheter as any break in the system carries the risk of introducing infection.

The diagnosis of peritonitis is based on the number of white blood cells found on microscopy alone. To make the diagnosis, the dialysis effluent white cell count should be $>100 / \mu \mathrm{L}$ or $>0.1 \times 10^{9} / \mathrm{L}$ (after a dwell time of at least 2 hours), with $>50 \%$ polymorphonuclear. Bacteria are present in low concentrations in dialysis effluent, so culture can be negative (approximately 20\% cases). The yield of positive cultures is increased by inoculating fluid into blood culture bottles. Positive cultures, in the absence of white blood cells on microscopy, usually represent contaminants and should therefore not be treated.

\section{Treatment of Peritonitis: Principles}

Treatment with antibiotics should be commenced as soon as possible in all patients with abdominal pain and cloudy fluid as soon as effluent fluid has been collected for microscopy and culture. If the fluid is only slightly hazy and the patient is otherwise well, it is reasonable to wait for the microscopy results. Peritonitis should be treated with intraperitoneal antibiotics. The advantages are:

- high antibiotic concentration in peritoneum;

- no intravenous access needed;

- patients can administer antibiotics themselves, minimizing need for hospitalization.

Intraperitoneal antibiotics are systemically absorbed and blood levels of potentially toxic antibiotics such as vancomycin and aminoglycosides need to be monitored. Numerous different antibiotic regimens have been developed in different hospitals. There is no evidence that one is any better than another as long as the following rules are followed:

- initial antibiotics with broad Gram-positive and Gram-negative cover;

- peritoneal concentration of antibiotic is high enough to eradicate infection (particularly important with oral antibiotics);

- follow-up of culture results and appropriate adjustments made to antibiotics;

- allowance for renal excretion of antibiotics if there is residual kidney function;

- measurement of vancomycin and aminoglycoside blood levels (as appropriate) to avoid underdosing if patients have residual kidney function, and overdosing with the potential side-effects of nephrotoxicity and ototoxicity;

- sufficient duration of treatment to avoid recurrence of infection;

- ease of administration, especially by patients, to avoid need for admission to hospital.

Patient-friendly regimens are based on once daily addition of antibiotics to dialysate. Nursing staff can add the antibiotics, and as the antibiotics concerned are stable for at least $48 \mathrm{~h}$ in solution, patients can collect three bags at a time for use at home. This regimen 


\section{Peritonitis in CAPD}

can also be used for patients on APD by adding antibiotics to a bag that is given when the patient comes off the machine and then allowed to dwell for 6 hours.

All peritoneal dialysis units should have a protocol for empirical antibiotics to be used as soon as there is a high suspicion of peritonitis. The 2016 ISPD guideline states:

- We recommend that empirical antibiotic therapy be initiated as soon as possible after appropriate microbiological specimens have been obtained (1C).

- We recommend that empirical antibiotic regimens be center-specific and cover both gram-positive and gram-negative organisms (1C).

- We recommend that gram-positive organisms be covered by vancomycin or a first generation cephalosporin and gram-negative organisms by a third-generation cephalosporin or an aminoglycoside (1B)

Culture results should be available by day 3-4. Antibiotics should then be adjusted as needed depending on organism identified and antibiotic sensitivities. Table 1 gives a useful summary for common organisms. The ISPD guideline should always be consulted for recommendations of antibiotics and dosages.

Figure 2: Flow diagram for early management of peritonitis

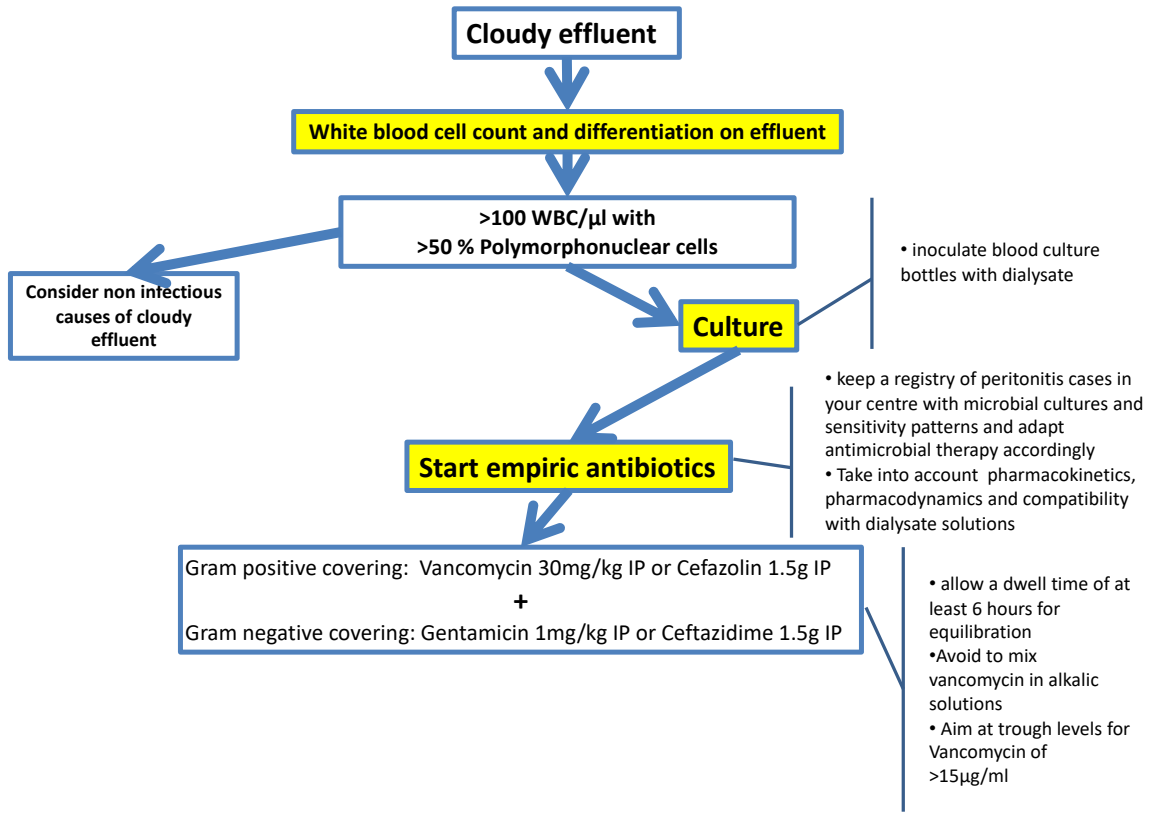

Table 1: Management of peritonitis according to causative organism

\begin{tabular}{|c|c|c|}
\hline Organism & Treatment & Duration \\
\hline $\begin{array}{l}\text { Staphylococcus } \\
\text { epidermidis }\end{array}$ & $\begin{array}{l}\text { Stop gentamicin. Continue Vancomycin 30mg/kg IP every 5-7 days for } 2 \text { weeks - } \\
\text { frequency of dosing depends on residual kidney function; aim for trough level } \\
>15 \mathrm{ug} / \mathrm{ml}\end{array}$ & 14 days \\
\hline $\begin{array}{l}\text { Staphylococcus } \\
\text { aureus }\end{array}$ & $\begin{array}{l}\text { Stop gentamicin. Continue Vancomycin } 30 \mathrm{mg} / \mathrm{kg} \text { IP every } 5-7 \text { days for } 3 \text { weeks - } \\
\text { frequency of dosing depends on residual kidney function; aim for trough level } \\
>15 \mathrm{ug} / \mathrm{ml} \text {. }\end{array}$ & 21 days \\
\hline $\begin{array}{l}\text { Pseudomonas } \\
\text { spp }\end{array}$ & $\begin{array}{l}\text { Stop Vancomycin. Will need } 2 \text { antibiotics depending on microbiology results. } \\
\text { Ceftazidime IP and oral ciprofloxacin useful } 2^{\text {nd }} \text { antibiotics. Gentamicin, if } \\
\text { continued, will need careful monitoring of blood levels to avoid nephro- and } \\
\text { ototoxicity }\end{array}$ & 21 days \\
\hline $\begin{array}{l}\text { Gram negative } \\
\text { infections }\end{array}$ & $\begin{array}{l}\text { Stop Vancomycin. Choice of antibiotics will depend on microbiology results. Will } \\
\text { often need two antibiotics. }\end{array}$ & 21 days \\
\hline Fungal & $\begin{array}{l}\text { Start anti-fungal treatment dependent on microbiology advice. REMOVE PD } \\
\text { CATHETER }\end{array}$ & \\
\hline $\begin{array}{l}\text { Culture } \\
\text { negative }\end{array}$ & $\begin{array}{l}\text { Stop gentamicin. Continue IP Vancomycin (see above) with oral Ciprofloxacin } \\
500 \mathrm{mg} \mathrm{bd}\end{array}$ & 14 days \\
\hline
\end{tabular}

\section{Treatment of automated peritoneal dialysis peritonitis}

- Patients can continue on usual APD regimen with antibiotics added to daytime exchange.

- There have been concerns that patients may present late, as cloudy fluid could be missed, as the PD fluid drained from machine is much more dilute than in CAPD (larger volumes). This might be a problem particularly if overnight fluid is drained directly into waste (e.g. a drain) and not into a bag. 


\section{Peritonitis in CAPD}

- Cure rates of APD peritonitis are same as for CAPD (i.e. about 80\%)

- Use of rapid exchanges may affect antibiotic pharmacokinetics, with need to change (increase) dosage.

\section{Treatment of Resistant Peritonitis}

Peritonitis failing to respond to treatment

Patients should symptomatically feel better, and fluid should be clear, within $2-3$ days of starting treatment. If not:

- Check antibiotic sensitivities and re-culture fluid.

- Consider removal of PD catheter if:

patient remains symptomatically unwell, i.e. remains febrile or no improvement in abdominal pain;

evidence of increasing sepsis, i.e. hypotension, fever, etc.;

fluid fails to clear despite appropriate treatment for 4days;

fluid becomes increasingly turbid;

catheter becomes blocked by thick fluid;

exit site infection or tunnel infection due to same organism as peritonitis;

peritonitis has become recurrent, i.e. repeat infection due to same organism within 4 weeks of original infection.

\section{Removal of PD catheter}

- This should not be delayed beyond 4 days from onset of non-responsive peritonitis as delay results in increased morbidity after catheter removal.

- Usually a simple procedure, but may need a general anaesthetic to make sure that dacron cuffs are fully removed

- Consider laparotomy if increasing sepsis, or in presence of 2 or more Gram-negative organisms, suggesting a possible bowel lesion.

\section{Reinsertion of PD catheter}

Many patients want to restart PD even after a severe episode of peritonitis requiring catheter removal. Patients may have poor vascular access necessitating a further attempt at PD.

- Allow at least 4 weeks before reinserting catheter.

- The catheter should be inserted surgically or laparoscopically because of the risk of adhesions,

- Retrain patient when recommencing PD to ensure there are no technique problems.

\section{Complications of Peritonitis}

Peritonitis remains a major cause of dropout from PD to HD. 20\% patients with peritonitis do not respond to antibiotic treatment.

Even if the episode has been fully treated without catheter removal, long-term morbidity can result:

- poor nutrition due to the catabolic state associated with peritonitis;

- loss of ultrafiltration during and after an episode;

- repeated episodes of peritonitis can cause long-term ultrafiltration failure necessitating transfer to haemodialysis;

- use of broad-spectrum antibiotics can cause diarrhoea from Clostridium difficile infection;

- fungal peritonitis may develop after repeated courses of intraperitoneal antibiotics;

- intra-abdominal adhesions making further PD difficult;

- ileus, fluid collections, and/or abscess formation, especially if catheter removal is delayed;

- death - there is a mortality rate of $2-5 \%$ with peritonitis, particularly in patients with multiple comorbidities.

\section{Summary}

Infection remains a major cause of concern for both healthcare teams and patients on any type of dialysis. With the development of protocols focusing on prevention and management of these infections, the risk of developing an infection can be greatly reduced and the cure rate can be greatly increased. Such protocols should be developed using the readily accessible ISPD guidelines and should be made easily accessible to all those involved with the management of patients on peritoneal dialysis.
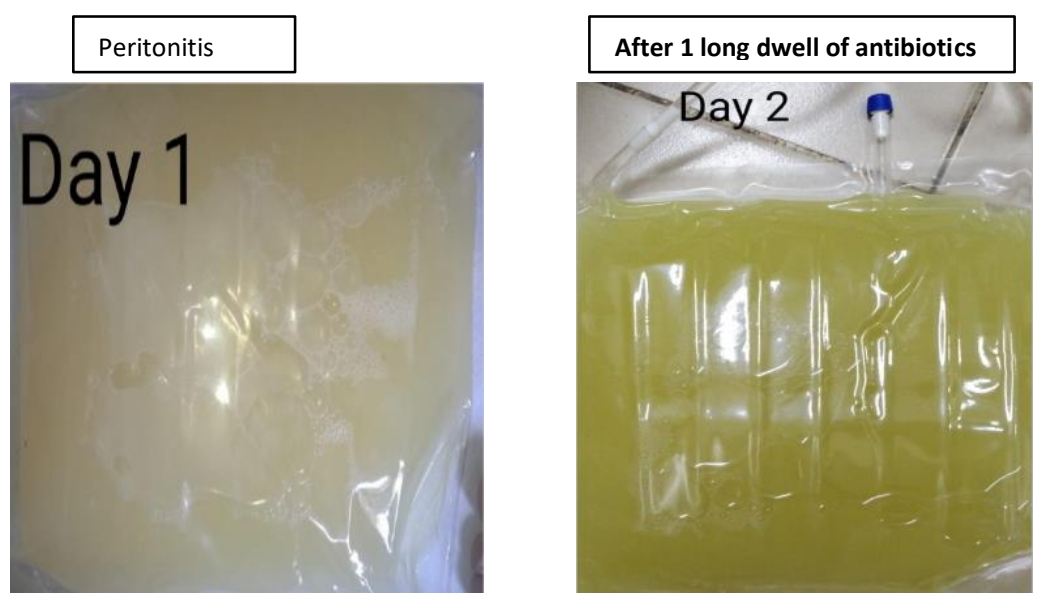